Pure \& Appl. Chem., Vol. 57, No. 3, pp. 515-522, 1985.

Printed in Great Britain.

(C) 1985 IUPAC

\title{
Luminescence measurements on surfaces
}

\author{
J. N. Miller \\ Department of Chemistry, Loughborough University of Technology, \\ Loughborough, Leicestershire, LE11 3TU, United Kingdom
}

\begin{abstract}
The paper describes the applications of solid surface luminescence measurements in the fields of cryogenic and room temperature phosphorescence, fluorescence immunoassays, and other areas. In many examples highly specific and sensitive assays are obtained by combining luminescence measurements with thin layer chromatography, and other laminar methods such as electrophoresis. Thin layer phosphorimetry is a promising approach, and may encourage the development of phosphorescent labels analogous to the currently common fluorescent labels. Several useful qualitative applications of surface luminescence have also been described, including the identification of glass samples, and the inclusion of phosphorescent and fluorescent markers on postage stamps and related materials.
\end{abstract}

\section{INTRODUCTION}

Photoluminescence and chemiluminescence methods of analysis have achieved widespread acceptance in the scientific community, particularly in the environmental and biomedical sciences. The well-known sensitivity of the methods is a crucial asset in these fields, but the two other advantages of luminescence spectrometry are just as important. Alone or in combination with other techniques, luminescence measurements can be relatively selective, and they have the further great advantage that they can be made with a wide variety of sampling arrangements. In addition to the conventional dilute solutions, concentrated solutions, suspensions, flowing liquids and solid surfaces can all be studied with ease. In this respect, luminescence spectrometry is greatly superior to most other spectrometric methods. This paper reviews some biomedical and other applications of solid-surface luminescence methods and similar technologies. The related topic of analytical selectivity features in many of the applications, and interesting experimental techniques and apparatus are also described.

Surface luminescence studies may be undertaken for a variety of reasons (Table 1), which may be classified into two groups. In some cases practical convenience will predominate - for example the desire to use very small volumes $(<=1 \mu 1)$ of samples and reagents, to study many samples in rapid succession, or to combine luminescence methods with chromatography or electrophoresis. In other instances surface measurements will have a more fundamental purpose, such as the observation of 'new' luminescence phenomena, or the protection of an analyte from quenching and other interactions.

TABLE 1. Surface luminescence studies

Study of microlitre sample volumes (e.g. luminescence sensors)

Analytical use of immobilised biochemical reagents (e.g. solid phase immunoassays)

Combined separation-luminescence methods (e.g. TLC-fluorimetry, TLC-phosphorimetry, electrophoresis-fluorimetry)

Rapid study of multiple samples (e.g. TLC/electrophoresis-luminescence)

Sensitised luminescence (e.g. energy transfer between neighbouring adsorbed molecules)

Protection of luminescent molecules from quenching (e.g. room temperature phosphorimetry) 
TABLE 2. Substrates for surface luminescence studies

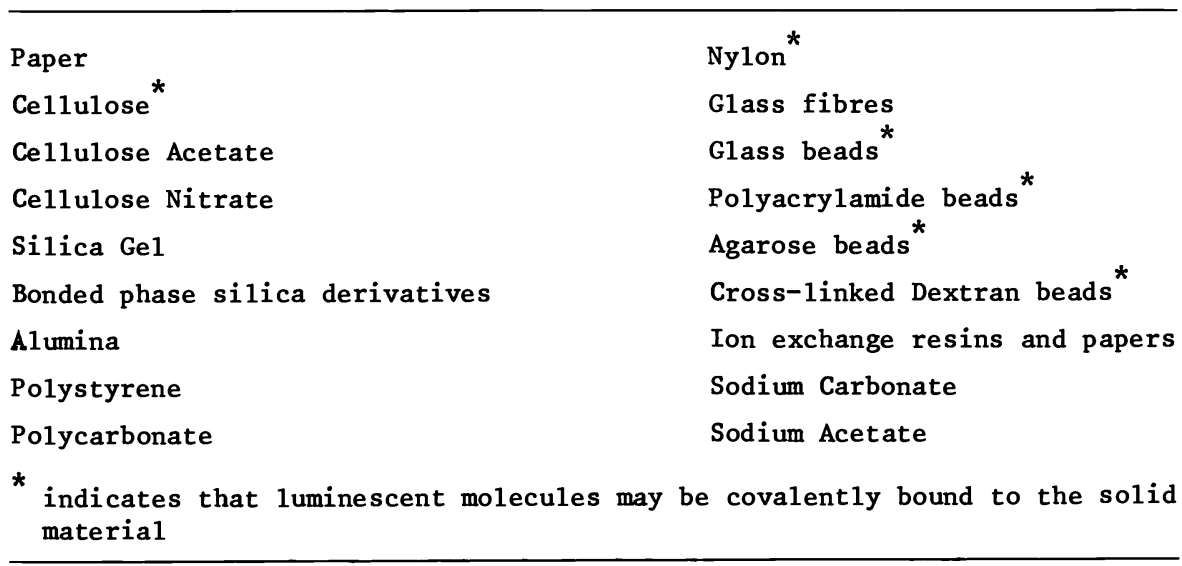

Quantitative relationships between the luminescence signal and the analyte concentration are more complex in surface luminescence analysis than in dilute solution work. All the numerous theoretical treatments (1-3) make fairly drastic, even unrealistic, assumptions about the nature of the solid medium and the optical conditions. So it is some comfort that most models predict that luminescence intensity will increase linearly with analyte concentration at low levels of analyte: this prediction has been borne out in numerous studies. The emitted photoluminescence can in principle be measured in both reflectance and transmission modes (4): the former is much the more common, except in some studies of transparent electrophoresis media.

Frequently, surface luminescence measurements can be performed by making only minor modifications to conventional fluorescence spectrometers or spectrodensitometers, but in other cases purpose-built equipment is required. New technologies such as diode array and related detectors (5), and fibre optics (6) and other miniature optoelectronic components, are already appearing in this field. Current interest in 'sensors' - small, convenient, even disposable analytical devices for use in special locations or conditions - is intense, and solid surface luminescence measurements will certainly play a role in their development. In most experiments, the analyte is placed on a surface such as chromatography paper, an electrophoresis membrane or gel, or a thin layer chromatography plate (Table 2), but other surfaces have been used, and there is scope for considerable development in this area. The observed luminescence denends a great deal on the distribution of the analyte in the thickness of the solid material, and on the presence of other solutes, and many selective and sensitive analyses have been based on the control of these variables.

\section{PHOSPHORIMETRY ON SOLID SURFACES}

Combination of fluorimetry and thin-layer chromatography (TLC) are commonplace in analytical work, and it is not surprising that considerable effort has been devoted to the study of phosphorescence on solid surfaces. For many years after its development as an analytical method (7), phosphorimetry was performed almost exclusively at a temperature of $77 \mathrm{~K}$, to minimise the bimolecular quenching interactions (especially those involving oxygen) commonly believed to make room temperature phosphorescence (RTP) a rare phenomenon.

Most of the first measurements of surface phosphorescence from organic molecules were thus also made in cryogenic conditions.

In the early 1970s the technique of thin layer phosphorimetry was developed in the author's laboratory $(8,9)$. This method allowed the determination of phosphorescence from solutes on a flexible solid substrate (e.g. a precoated TLC plate) cooled by conduction to liquid nitrogen temperature. The compact apparatus, designed as an accessory for a range of fluorescence spectrometers and incorporating a single disc phosphoroscope, also permitted the atmosphere immediately above the solid surface to be controlled, so that (for example) the formation of ice crystals at low temperatures could be prevented. Many therapeutic drugs could be determined phosphorimetrically at nanogram levels, and the combination of chromatographic separation and carefully chosen excitation and emission wavelengths could generate highly selective analyses. A precise and accurate method for the determination of plasma phenytoin levels (Fig. 1), and the analysis of mercaptopurine drugs (10) and polynuclear hydrocarbons (PAHs) are more recent examples of the application of this approach. Greatly enhanced sensitivity is obtained if the surface of the TLC plate is sprayed with a suitable solvent immediately before the phosphorescence analysis (Table 3). This is doubtless due in part to a concentration of the sample molecules at the surface of the plate, but more specific interactions are probably also involved (11). 
Plasma + Phenytoin

Extract at $\mathrm{pH} 6.8$

(1,2 dichloroethane)

$\downarrow$

Permanganate oxidation

$\downarrow$

Benzophenone

$\downarrow$

Extraction (methylcyclohexane)

\section{$\downarrow$}

TLC

(Silica gel: chloroform/methanol/acetone, $6 / 1 / 1 \mathrm{v} / \mathrm{v} / \mathrm{v}$ )

$\downarrow$

Phosphorimetry

(Excitation $260 \mathrm{~nm}$; Emission $446 \mathrm{~nm}$ )
TABLE 3. Enhanced phosphorescence emission from drugs adsorbed gel TLC plates at $77 \mathrm{~K}$

\begin{tabular}{clc}
\hline Substance & $\begin{array}{l}\text { Sprayed } \\
\text { solvent }\end{array}$ & $\begin{array}{c}\text { Enhancement } \\
\text { factor }\end{array}$ \\
\hline Phenobarbitone & Methano1 & 15 \\
" & Ethano1 & 20 \\
" & 1-Butano1 & 34 \\
N-methy1 phenobarbitone & Ethano1 & 16 \\
5-pheny1 5-methy1 & Ethano1 & 18 \\
barbituric acid & Methano1 & 65 \\
Sulphadiazine & Ethano1 & 91 \\
" & 1-Butano1 & 100 \\
" & Ethano1/KI & 205 \\
" & Ethanol & 165 \\
Sulphamerazine & Ethanol & 240 \\
Sulphamethazine & Ethano1 & 55 \\
Pericyazine & " & 52 \\
Chlorpromazine-HC1 & & \\
& &
\end{tabular}

Limit of detection $0.4 \mathrm{ng}$; Recovery $98.3 \%$ Linear Range 0.5 - $45 \mathrm{ng}$

Fig. 1. Determination of plasma phenytoin by thin layer phosphorimetry.

Great interest was aroused by the 'discovery' about 15 years ago that room temperature phosphorescence (RTP) could be observed from a range of molecules adsorbed on solid surfaces (12). Subsequent researches (13) showed that the phenomenon had actually been detected some 25 years earlier (14): but only in the 1970s was it studied intensively as an analytical method. Early suggestions that only anionic species could be studied were rapidly dispelled, and over 200 substances, many of biochemical interest, have been analysed by the RTP method (Table 4). Enhanced RTP signals, and additional analytical selectivity, were found to be obtained by the introduction of heavy-atom perturbants and/or detergent micelles $(15,16)$. A feature of many early studies was that, while complete absence of water vapour seemed to be necessary for observations of RTP, oxygen quenching was of less consequence. This led to the suggestion that hydrogen bonding was an important mechanism for the adsorption of the analyte on to the solid surface (17). Any water present would naturally compete for the same binding sites on the solid surface. In practice, however, so many analytes and so many types of surface have been used to produce analytically useful RTP phenomena, that any single, simple mechanistic explanation for the phenomenon seems unlikely. Only recently $(18,19)$ have serious attempts been made to use other spectroscopic methods to study the state of the adsorbed molecules: it seems likely that photoacoustic spectrometry may have considerable value in this connection.

Superficially, the RTP technique seems to have every advantage. It avoids the inconvenience of measurements at $77 \mathrm{~K}$, while retaining the other advantages of phosphorimetry. In practice, however, it must be regarded as only a partial success (Table 5). In many cases the RTP signal is at least an order of magnitude less than the phosphorescence intensity at $77 \mathrm{~K}(20)$. Moreover there is often considerable background luminescence from the solid support so the limits of detection of the method are only moderate. Careful selection of the experimental conditions (solid support, heavy atom perturber, possible use of surfactants, etc.) may be necessary before any useful RTP occurs, and the need to remove water vapour complicates the experimental set-up. RTP spectra are often broader and displaced to longer wavelengths than their $77 \mathrm{~K}$ counterparts: the band broadening increases spectral overlaps and adversely affects the selectivity and the detection limits of RTP methods. 
Some techniques, already applied in conventional fluorimetry, are available to tackle the problems of spectral overlap and solid matrix background emission. Most easily applied among these are synchronous scanning spectrometry, and derivative spectroscopy. Synchronous spectra (21) are obtained by the simultaneous scanning of both the monochromators of the fluorescence spectrometer, with a fixed (or even a variable) wavelength difference between them. Correct choice of this wavelength interval can greatly reduce the spectral bandwidth of the phosphorescence signal, effectively discriminating against overlapping spectra. Derivative spectra (22), easily obtained by optical or electronic accessories or by calculation, also cause a reduction in spectral bandwidths and discriminate in favour of sharp bands and against broad background signals. Both these methods are of value in RTP studies, and they may be used in combination - derivative synchronous spectra yield very narrow bandwidths indeed (23). Other possible developments in the RTP technique include automated background subtraction (24) and photochemical background reduction methods (25); the use of time resolution with a pulsed source phosphorimeter (though it must be noted that almost all RTP lifetimes are very short); new approaches to improving the precision of RTP analyses (26); and the use of new surfaces and new techniques such as the incorporation of metal colloidal solutions with the sample (27). In addition the combination of RTP methods and TLC should be very powerful. This hybrid method has so far been little used (26-28), perhaps because the solvents used for the TLC separation are not suitable for subsequent RTP measurements. The success of similar experiments at $77 \mathrm{~K}(8,9)$, and the possible development of phosphorescent or phosphorigenic labels (29) (Fig. 2) analogous to those used in fluorimetry suggest that TLC-RTP measurements in solution - particularly those using

TABLE 4. Some classes of compounds studied by room temperature phosphorimetry

Aromatic hydrocarbons

Substituted aromatic systems

Nucleosides

Nucleotides

$\mathrm{N}$-heterocycles

Substituted phenols and naphthols

Barbiturates

Sulphonamides

Mercaptopurines

Aromatic dyes

Aromatic Amino-acids TABLE 5. Advantages and disadvantages of RTP on solid surfaces<smiles>O=c1ccc2cc(S(=O)(=O)Cl)ccc2o1</smiles><smiles>CSc1cc(C#N)ccc1C1(c2cc(Br)c(O)c(Br)c2Oc2nc(Cl)nc(Cl)n2)OC(=O)c2cc(Br)c(O)c(Br)c2Oc2c1cc(Br)c(O)c2Br</smiles>

Fig. 2. Structures of some phosphorescent

Advantages

Disadvantages

Simpliticy (no liquid $\mathrm{N}_{2}$ )

Selectivity (different heavy atom perturbants and solid substrates)

Additional luminescent compounds (non-fluorescent compounds)

Potential for TLC-phosphorimetry
Removal of water vapour

Lower sensitivity than cryogenic phosphorimetry

Trial and error selection of substrate and heavy atom perturbant

Background luminescence from solid surface affects limits for direction

Short phosphorescence lifetimes make time-resolved phosphorimetry problematical 
surfactants or cyclodextrins (30) to protect the sample from quenching interactions - seem to be attracting greater attention. All aspects of RTP have recently been surveyed in a use ful monograph (31).

\section{IMMUNOASSAYS USING SOLID SURFACES}

The development of fluorescence immunoassays has proceeded rapidly in recent years, and several systems are now commercially available. Much research has involved the development of homogeneous assays (32), which make use of a change in fluorophore properties accompanying antibody binding of a fluorescent labelled analyte, and hence need no separation step to discriminate between antibody bound and unbound molecules. Such assays are in theory very convenient to perform and easily automated, but in practice they suffer from severe limitof-detection problems because of background interferences from endogenous fluorophores in blood plasma and other likely samples.

Many systems still thus use solid phases to separate antibody bound and unbound materials, and this may involve fluorescence (or chemiluminescence) measurements on solid surfaces. Although such systems allow the removal in simple washing steps of much of the fluorescence background background signal, new problems of background scattered light and fluorescence from the solid surface inevitably appear. Careful instrument design (and choice of fluorophore and solid surface) is thus necessary. Fibre optic fluorescence spectrometers are bound to be of particular value: they can be used to illuminate a very small area of a solid surface (so minimising the use of costly reagents and precious samples), and the angles of incidence and observation can easily be controlled to maximise signal: background ratios.

A recent design exemplifies these advantages (33). The instrument (which can also be used for diffuse reflectance measurements) is used to study a solid phase version of the Substrate Labelled Fluorescent Immunoassay (SLFIA - Fig. 3): this method was, ironically, designed as a homogeneous assay, but it has been successfully adapted to solid phase technology. The main problem is that the solid phase (chromatography paper with a reflective Mylar backing) must be uniformly impregnated with the reagents in such a way that they do not interact before addition of the sample. In practice this is achieved (34) by using two different solvent systems, with separate application of (a) the antibodies, enzyme and an aqueous buffer, and (b) an acetone solution of the substrate labelled analyte. Impregnation is performed by soaking the paper completely in each solution, and the paper is dried at $50^{\circ} \mathrm{C}$ after each step. The precision of the measurements is very high, and the analysis of each sample is completed within 3 minutes. It seems certain that, if relatively complex procedures of this kind can be performed using impregnated paper as the reagent and sample holder, many other assays could be similarly adapted. The spectrometer is optically very simple and robust, yet remains capable of nanomolar detection limits in therapeutic drug assays.

Another extremely ingenious immunoassay system relies on the concentration dependent migration rate of an analyte through a solid phase (filter paper) impregnated with antibody. The solid surface is coated with antibody, and then contacted with the sample. The greater the antigen concentration in the sample, the more it will penetrate into the solid support. The unused antibody binding sites are then reacted with fluorescent labelled antigen, and. the amount of label bound at the centre of the solid support measured (35). A variant of the method uses enzyme-labelled antigen and a fluorigenic substrate at the measurement stage.

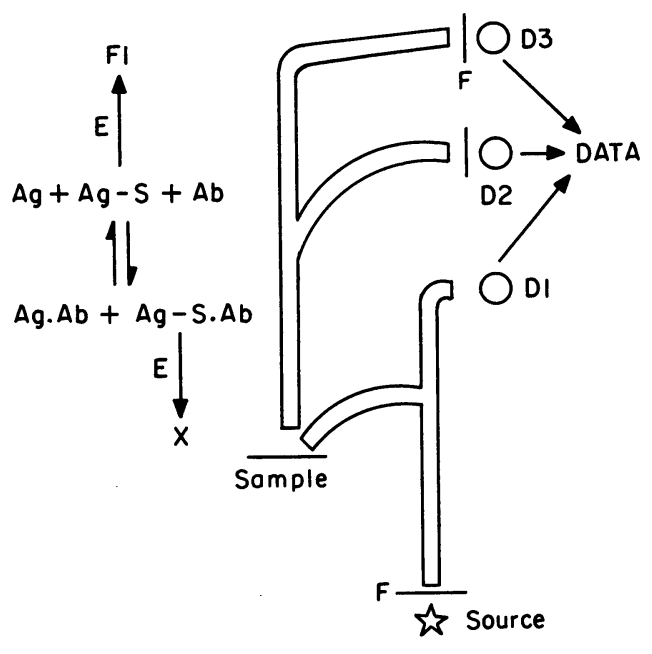

Fig. 3. Substrate labelled fluorescence immunoassay performed using immobilised reagents and a fibre optic spectrometer: F-filters, D-detectors. 
Solid phase chemiluminescence (CL) immunoassays have also been developed, and may be expected to have the advantages of sample and reagent economy, and relative freedom from interferences. In addition, CL instrumentation is simpler and often vastly more sensitive than photoluminescence equipment. Little information is available on the background CL of solid phases, but some early assays show great promise. An interesting example is the use of hemin as a catalytic label in the determination of albumin (Fig. 4). Hemin labelled albumin bound to immobilised antibody catalyses the luminol CL reaction after removal of possible interferences in a washing step (36).

Solid phase methods will clearly continue to be widely used in luminescence immunoassays. In some cases the motivation will be the simple advantages of background removal and reagent and sample economy. In addition, assays in which the solid phase plays a direct role in the photochemical process may become more popular. Examples include the total internal reflectance assay method (37), and fluorescence fluctuation immunoassay (38).

\section{OPTICAL SENSORS BASED ON SOLID SURFACE LUMINESCENCE}

It will be clear from the preceding examples that solid phase luminescence measurements have many merits, even when made with fairly conventional equipment and familiar chemical systems. There are many instances, however, where the use of new instrumentation or unusual photochemical principles will bring additional benefits.

The best example of this is perhaps the use of immobilised reagents in fibre optic sensors. The fluorescence of immobilised molecules has been measured on several occasions, for example in studies of molecular conformations. A new approach, using the fluorescence of immobilised species for analytical purposes, is now developing. These new devices are the natural offspring of the remote sensing "optrodes" of Hirschfeld. He demonstrated that iodine could be determined using its quenching effect on rubrene (39): the PAH was contained in an insoluble polystyrene gel illuminated and observed by an optical fibre. The general principle of the simplest fibre optic sensors, illustrated in Figure 5, involves the measurement of enhanced or quenched fluorescence (or, in principle, phosphorescence or chemiluminescence) accompanying the binding of the analyte to an immobilised reagent. Other applications of this principle have included the determination of metal ions (40), oxygen $(41,42), \mathrm{pH}(43)$, and halide ions (44). Many of these analyses are based on well established fluorescence quenching interactions. Thus, halide ions are determined using their quenching effects on quinolinium compounds, and oxygen by its quenching action on perylene or pyrene derivatives. A sensor for $\mathrm{pH}$ has been based on the fluorescence properties of fluorescein, and metal ions ( $\mathrm{Al}^{3+}, \mathrm{Mg}^{2+}$ etc.) can be analysed with the aid of the greatly enhanced fluorescence they yield with such chelating ligands as morin or 8-hydroxyquinoline sulphonic acid. These reagents have been immobilised by covalent linkages or ion exchange interactions on such surfaces as glass, beaded ion exchange resins or membranes. All the sensors are characterised by extreme simplicity and robustness. Very compact light sources such as light emitting diodes, sample detectors such as photodiodes, and cheap plastic fibre optic cables can be used.

\section{SURFACE CLIA} Uses hemin catalysis of luminol CL reaction

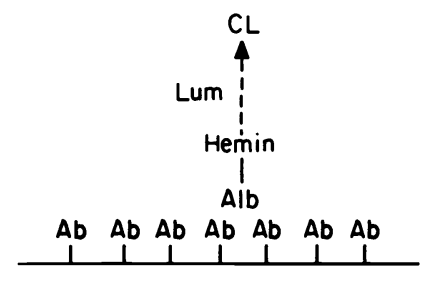

$$
\text { Lum - luminol Alb-albumin }
$$

Fig. 4. Solid surface chemiluminescence immunoassay for serum albumin.

\section{FIBRE OPTIC SENSORS}

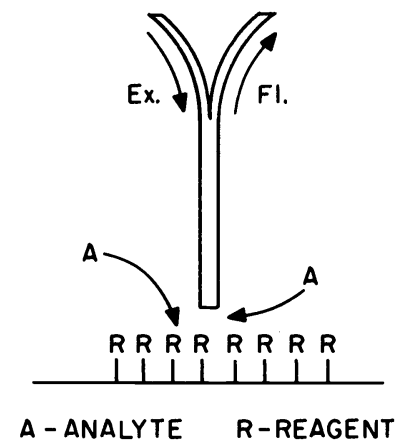

Fig. 5. Fibre optic sensor design for solid phase luminescence measurements.

The potential of such systems in biochemical analysis is clearly immense, and optical sensors have a number of advantages over the electrochemical sensors mainly studied so far. Two important developments are foreshadowed by recent papers. In one case the potential for multi-wavelength (and hence in principle multi-analyte) determinations was demonstrated in a $\mathrm{pH}$ sensor based on the fluorescence properties of the neutral and ionised forms of immobilised 8-hydroxypyrene-1,3,6-sulphonic acid (45). Dual wavelength measurements have the obvious additional advantage of controlling fluctuations in light source intensity, temperature effects etc. In another important development a sensor for glucose was based on a competitive binding interaction between the analyte and fluorescein-labelled dextran, the receptor being immobilised concanavalin A (46). Since methods for the immobilisation of 
antibodies on many types of surface are well established, it can hardly be doubted that similar sensors for monitoring immunoassays will be perfectly feasible. Optical sensors have been recently reviewed (47).

\section{OTHER SOLID SURFACE LUMINESCENCE MEASUREMENTS}

In this section a number of other recent advances in the field of surface luminescence measurements are surveyed, and some possible developments are foreshadowed. Overall, it seems certain that such methods will increase in importance. It is possible also that similar experimental arrangements will become more popular in the fluorimetry of dilute solutions: the designers of a recently described fibre optic fluorescence spectrometer (48) have re-emphasised the advantages of 'front-face' optics for the study of dilute solutions. Inner filter artefacts are abolished, and flowing as well as static samples can be studied.

The use of lasers as light sources in fluorescence spectrometry may have been limited by their high cost. There are also technical difficulties, notably the likely photodecomposition of the sample and the fact that laser sources may increase background interference signals as well as the desired analyte signal. But it is evident that laser excitation has great potential in the analysis of small areas of solid surfaces. One important benefit is the ability to excite luminescence phenomena unobtainable with conventional sources. In particular, two-photon excitation processes are feasible, and have the great merit that the wavelength of the emitted fluorescence is shorter than the excitation wavelength. This so greatly reduces the background signal that excellent limits of detection are available even for processes with low efficiencies. Thus subnanogram detection limits for fluorescent derivatives of amines, and for various scintillants, have been claimed using two photon excitation processes with submicrolitre samples on a reversed phase high performance TLC (HPTLC) plate (49). A dye laser pumped by a nitrogen laser was the light source. The combination of laser sources and HPTLC plates will be especially appropriate in view of the small sample zones obtained.

Another well-established photochemical technique is the generation of sensitised fluorescence. In this method, light is absorbed by one fluorophore, present at reasonably high concentrations, and the energy is transferred non-radiatively to the analyte fluorophore, presumed to be present at trace levels. In this way the trace component can often be excited more efficiently than is possible by direct illumination. Such phenomena are commonplace in solution, and have been the basis of many studies of molecular interactions, as well as of fluorescence immunoassays and enzyme assays. But it has been pointed out that energy transfer efficiencies are often particularly high in solid media, so "surface sensitised luminescence" may be a simple and very sensitive trace analysis method. Thus far it has been applied only to the analysis of hydrocarbons (50), but there seems to be no reason why other molecules should not be detected in this way. Solid surface energy transfer immunoassays, for example, might prove to be more sensitive and less prone to interferences than the corresponding solution assays.

Still another largely unexplored area is that of spectroelectrochemistry. A number of experiments have shown that fluorescent or chemiluminescent species can be efficiently generated at the surfaces of various electrodes. The observation of such species is clearly similar to a solid surface luminescence experiment. The electrochemical conditions provide an extra element of selectivity for the analysis, and permit the production of transient and unstable species $(51,52)$. Sample cells suitable for the study of fluorescent materials generated electrochemically have been described (53), but this technique remains to be fully exploited.

The extraordinary sensitivity of chemiluminescence (CL) measurements has aroused great interest in biochemical analysis, but few applications of the method to the monitoring of TLC separations have been described. Perhaps the best approach (54) to this problem is the well known oxalate CL reaction, which generates excited fluorophore molecules via energy transfer from an oxetanedione intermediate. The method has the advantage that a very large range of fluorophores can be excited: moreover, the experimental conditions can be controlled by the user to yield bright but short-lived or feebler, long-lived light emissions. Given again the efficiency of energy transfer processes on solid materials, this technique should find wider use.

Finally it is worth recalling that in the United Kingdom and other countries, most postage stamps are printed on fluorescent or (usually) phosphorescent paper: and letters and other postal items are often coded with luminescent marks to help the mail sorting process (55, 56). This application is very far from the biochemical laboratory, but it is instructive in many ways. It indicates that a wide variety of papers and other materials coated with fluorescent or phosphorescent substances are readily available; shows that phosphorescence measurements are entirely feasible on a large scale and in many environments; and points the way to the use of phosphorescent or fluorescent tags in laboratory management systems, for example for the labelling or coding of sample containers of different types. It is clear that screening tests and other qualitative or semi-quantitative analyses incorporating luminescent labels could be performed at high sample throughput rates, without the use of 
skilled labour, and with the results used to regulate automatically the next test to be performed or the reporting of the results. Along with the more sophisticated methods described in earlier parts of this brief review, such developments will ensure that luminescence measurements on solid surfaces continue to play an important role in analytical chemistry and analytical biochemistry.

\section{REFERENCES}

1. G. Kortum, Reflectance Spectroscopy, Springer-Verlag, New York, (1969).

2. J. Goldman, J. Chromatogr. 78, 7-18 (1973).

3. V. Pollak, Optica Acta $21, \overline{51}-60$ (1974).

4. R.J. Hurtubise, Solid Surface Luminescence Analysis, Marcel Dekker, New York, (1981).

5. Y. Talmi, D.C. Baker, J.R. Jadamec and W.A. Saner, Anal. Chem. 50, 936A-951A (1978).

6. I. Chabay, Anal. Chem. 54, 1071A-1080A (1982).

7. R.J. Keirs, R.D. Britt Jnr. and W.E. Wentworth, Anal. Chem. 29, 202-208 (1957).

8. L.A. Gifford, J.N. Miller, D.T. Burns and J.W. Bridges, J. Chromatogr. 103, 15-23 (1975) .

9. J.N. Miller, UV Spectrometry Group Bulletin No. 7, 82-92 (1979).

10. A.I. Al-Mosawi, J.N. Miller and J.W. Bridges, Analyst 105,

11. J.N. Miller, D.L. Phillipps, D.T. Burns and J.W. Bridges, Anal. Chem. 50, 613-616 (1978).

12. E.M. Schulman and C. Walling, Science 178, 53-55 (1972).

13. J.B.F. Lloyd and J.N. Miller, Talanta $\overline{26}, 180$ (1979).

14. H.E. Millson, The Phosphorescence of Textile Fibres \& Other Substances, Calio Tech. Bulletin No. 753, American Cyanamid Co. (1944).

15. P.G. Seybold and W. White, Anal. Chem. 47, 1199-1201 (1975).

16. L.J. Cline Love, M. Skrilec and J.G. Habarta, Anal. Chem. 52, 754-759 (1980).

17. E.M. Schulman and R.T. Parker, J. Phys. Chem. $81,1932-1935$ (1977).

18. L.J. Cline Love, M. Skrilec and J.G. Habarta, Anal. Chem. 52, 754-758 (1980).

19. R.A. Dalterio and R.J. Hurtubise, Anal. Chem. $\overline{56,336-341} \overline{(1984)}$.

20. A.I. Al-Mosawi, Ph.D. Dissertation, Loughborough University of Technology, (1979).

21. T. Vo-Dinh, in Modern Fluorescence Spectroscopy, Vol. 4, (E.L. Wehry, ed.), p.167, Plenum Press, New York, (1981).

22. G.L. Green and T.C. O'Haver, Anal. Chem. 46, 2191-2196 (1974).

23. T. Vo-Dinh, App1. Spectroscopy 36, 576-581 (1982).

24. S.Y. Su, D. Bolton and J.D. Winefordner, Chem. Biomed. Environ. Inst. 12, 55-60 (1982).

25. D.L. McAleese and R.B. Dunlap, Anal. Chem. 56, 600-601 (1984).

26. M.W. Warren III, J.P. Avery and H.V. Malmstadt, Anal. Chem. 54, 1853-1858 (1982).

27. S.Y. Su and J.D. Winefordner, Can. J. Spectrosc. $\overline{28}, 21-29$ (1983).

28. C.D. Ford and R.J. Hurtubise, Anal. Chem. 50, 610-612 (1978).

29. E.U. Akusoba and J.N. Miller, Proc. Anal. Div. Chem. Soc. 16, 93-96 (1979).

30. S. Scypinski and L.J. Cline Love, Anal. Chem. 56, 322-327 (1984).

31. T. Vo-Dinh, Room Temperature Phosphorimetry for Chemical Analysis, Wiley-Interscience, New York, 1984

32. E.F. Ullman, N.F. Bellet, J.M. Brinkley and R.F. Zuk, in Immunoassays; Clinical Laboratory Techniques for the 1980s, (R.M. Nakamura et al., eds.), Alan R. Liss, New York, (1980).

33. W.E. Howard III, A. Greenquist, B. Walter and F. Wogoman, Anal. Chem. 55, 878-881 (1983).

34. B. Walter, A.C. Greenquist and W.E. Howard III, Anal. Chem. 55, 873-878 (1983).

35. C. Glad, Appl. Biochem. Technol. 7, 75-79 (1982).

36. Y. Ikarlyama, S. Suzuki and M. Aizawa, Anal. Chem. 54, 1126-1129 (1982).

37. M.N. Kronick and W.A. Little, United States Patent No. 3,939,350 (1976).

38. D.F. Nicoli, J. Briggs and V.B. Elings, Proc. Nat. Acad. Sci. USA 77, 4904-4908 (1980).

39. S.A. Borman, Anal. Chem. 53, 1616A-1618A (1981).

40. M.A. Ditzler, G. Doherty, S. Sieber and R. Allston, Anal. Chim. Acta 142, 305-311 (1982).

41. D.W. Lubbers and N. Opitz, in Proceedings of the International Meeting on Chemical Sensors, Fukuoka, Japan, p.609, Elsevier, Amsterdam, (1983).

42. J.I. Peterson, R.V. Fitzgerald and D.K. Buckhold, Anal. Chem. 56, 62-67 (1984).

43. L.A. Saari and W.R. Seitz, Anal. Chem. 54, 821-823 (1982).

44. E. Urbano, H. Offenbacher and 0.S. Wolfbeis, Anal. Chem. 56, 427-429 (1984).

45. Z. Zhujun and W.R. Seitz, Anal. Chim. Acta, in the press

46. J.S. Schultz, United States Patent No. 4,344,438 (1982).

47. W.R. Seitz, Anal. Chem. 56, 16A-34A (1984).

48. E.H. Ratzlaff, R.G. Harfmann and S.R. Crouch, Anal. Chem. 56, 342-347 (1984).

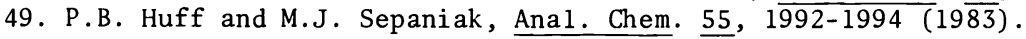

50. P.G. Seybold, D.A. Hinckley and T.A. Heinrichs, Anal. Chem. 55, 1994-1996 (1983).

51. A. Yildiz, P.T. Kissinger and C.N. Reilley, Anal. Chem. 40, $\overline{1018-1020 ~(1968) . ~}$

52. J.S. Pflug and L.R. Faulkner, J. Amer. Chem. Soc. 102, 6143-6144 (1980).

53. B.L. Cousins, J.L. Fausnaugh and T.L. Miller, Analyst 109, 723-726 (1984).

54. T.G. Curtis and W.R. Seitz, J. Chromatogr. 134, 343-350 (1977).

55. A. Walker, Philatelic Bulletin, June 1979, 4-6.

56. A. Walker, Philatelic Bulletin, July 1979, 5-8. 